\title{
Prediction of the difficulty of proximal vascular control using 3D-CTA for the surgical clipping of internal carotid artery-posterior communicating artery aneurysms
}

\author{
Takeya Niibo, MD,1 Katsumi Takizawa, MD, ${ }^{1}$ Jurou Sakurai, MD, ${ }^{1}$ Seizi Takebayashi, MD, PhD, ${ }^{1}$ \\ Hiroyasu Koizumi, MD, PhD, ${ }^{1}$ Toru Kobayashi, MD, PhD, ${ }^{1}$ Rina Kobayashi, MD, ${ }^{1}$ \\ Kouta Kuris, MD, PhD, ${ }^{1}$ Syusuke Gotou, MD, ${ }^{1}$ Ryousuke Tsuchiya, MD, ${ }^{1}$ and \\ Hiroyasu Kamiyama, MD²
}

1Department of Neurosurgery, Asahikawa Red Cross Hospital, Asahikawa; and 2Department of Neurosurgery, Sapporo Teishinkai Hospital, Sapporo, Japan

OBJECTIVE During surgical clipping of internal carotid artery (ICA)-posterior communicating artery (PCoA) aneurysms, proximal vascular control (PVC) is difficult to achieve in some cases because of variations in the anatomy of this type of aneurysm and its parent arteries. The authors investigated morphometric features that may be predictive for the necessity of anterior clinoidectomy (ACL) or cervical ICA exposure for PVC.

METHODS The authors retrospectively reviewed 65 patients with an ICA-PCoA aneurysm treated with clipping during the previous 3 years. The factors considered for assessing the difficulty of attaining PVC included the following: the maximum diameter of the aneurysm; the distance between the tip of the anterior clinoid process (ACP) and the proximal aneurysmal neck; the presence of calcification at the ophthalmic segment of the ICA; and the angles between the communicating segment of the ICA and the ophthalmic segment of the ICA and a line perpendicular to the cranial base, which reflect the tortuosity of the ICA. These parameters were measured based on preoperative CTA results.

RESULTS In a total of 21 patients (32.3\%), PVC was difficult to perform with the usual pterional approach. In 6 patients, temporary artery occlusions (TAOs) were difficult to achieve because of severe atherosclerotic wall changes in the ophthalmic segment of the ICA. For 15 patients, the ACPs overhanging the ophthalmic segment of the ICA obstructed the ability to secure a space for TAO. In the 21 patients with PVC difficulty, ACL alone, cervical ICA exposure alone, and both ACL and cervical ICA exposure were conducted in 6, 8, and 7 patients, respectively. Multivariate analysis with binary logistic regression revealed that the maximum diameter of the aneurysm $(p=0.041)$, the distance between the proximal neck of the aneurysm and the ACP tip $(p=0.002)$, and calcification of the ICA ophthalmic segment $(p=0.001)$ were significant predictive factors for difficulties with PVC. A receiver operating characteristic curve analysis revealed that a distance between the proximal aneurysmal neck and the ACP tip of $\leq 5.4 \mathrm{~mm}$ was the best cutoff value for predicting the difficulty of attaining PVC (area under the curve 0.800 , sensitivity $80.0 \%$, specificity $80.0 \%$ ).

CONCLUSIONS A short distance between the proximal aneurysmal neck and the ACP tip and the presence of calcification at the ophthalmic segment of the ICA on preoperative CTA are helpful for predicting the difficulty of achieving PVC.

https://thejns.org/doi/abs/10.3171/2020.1.JNS192728

KEYWORDS internal carotid artery; posterior communicating artery; aneurysms; anterior clinoid process; anterior clinoidectomy; vascular disorders

I NTRAOPERATIVE rupture (IOR) of an intracranial arterial aneurysm can dramatically interrupt a deliberate microsurgical procedure. In particular, a premature rupture before the establishment of proximal vascular control (PVC) can lead to catastrophic bleeding and jeop- ardize the patient's chances for a favorable outcome. The incidence of IOR during microsurgery has been estimated within a wide range from $7 \%$ to $40 \% .^{1-3}$ In the case of IOR, the reported associated mortality is also diverse, ranging from $0 \%$ to $33 \%$. $^{1,3}$ Temporary artery occlusion (TAO) of

ABBREVIATIONS $A C h A=$ anterior choroidal artery; $A C L=$ anterior clinoidectomy; $A C P=$ anterior clinoid process; $A P=$ anteroposterior; ICA = internal carotid artery; IOR = intraoperative rupture; $\mathrm{PCOA}=$ posterior communicating artery; $\mathrm{PVC}=$ proximal vascular control; ROC = receiver operating characteristic; $\mathrm{TAO}=$ temporary artery occlusion. SUBMITTED October 7, 2019. ACCEPTED January 27, 2020.

INCLUDE WHEN CITING Published online April 10, 2020; DOI: 10.3171/2020.1.JNS192728. 
the arteries proximal to an aneurysm reduces the pressure within the aneurysm, which may improve the safety of aneurysm dissection and reduce the risk of IOR and its associated mortality. ${ }^{4,5}$ Leipzig et al. reported that aneurysms treated without TAO had more than twice the rate of IOR than aneurysms treated with TAO. ${ }^{2}$

Internal carotid artery (ICA)-posterior communicating artery $(\mathrm{PCoA})$ aneurysms are more liable to rupture intraoperatively than aneurysms in other locations, and there is a lower rate of IOR in operations using TAO. ${ }^{2}$ Thus, securement of the proximal parent artery of an aneurysm in preparation for IOR is of paramount importance for a favorable outcome in clipping surgery of ICA-PCoA aneurysms. The proximal ICA of aneurysms can be secured with a usual pterional approach in most cases. However, the procedure is difficult in some cases because of variations in the anatomy of this type of aneurysm and its parent arteries. In particular, in cases where the anterior clinoid process (ACP) is overhanging the ICA at the proximal portion of the aneurysm, the removal of the ACP or cervical ICA is necessary for PVC. Although not all cases require these procedures for PVC, if the procedure is not used when necessary, the surgery will be more complicated. Therefore, preoperative prediction of the difficulty of establishing PVC is important in surgical planning for ICA-PCoA aneurysms.

In this study, we investigated the anatomical features that facilitate the prediction of the difficulty of attaining PVC and the need for anterior clinoidectomy (ACL) or cervical ICA exposure for PVC in surgical clipping cases of ICA-PCoA aneurysms.

\section{Methods \\ Patient Population}

During a 3-year period (January 2015 to December 2017), 65 patients with 45 ruptured and 20 unruptured PCoA aneurysms underwent surgical clipping at our institution and were enrolled in this retrospective study. All patients were diagnosed with an ICA-PCoA aneurysm based on intraoperative findings. Approval for the collection and review of data was obtained from the institutional review board of the Asahikawa Red Cross Hospital.

\section{Strategy for PVC in Surgical Clipping of ICA-PCoA Aneurysms}

All of the ruptured and unruptured ICA-PCoA aneurysm surgeries were conducted by a senior neurovascular surgeon (K.T.). After a standard pterional craniotomy, the dura was opened via a semicircular incision, revealing the sylvian fissure and the frontal and temporal lobes. The sylvian cistern was then opened at the level of the opercular frontal gyrus and dissected down to the carotid bifurcation. Through these manipulations, the optic nerve and the ICA could be observed. PVC was conducted in all patients. If securing a space for TAO was difficult due to an ACP coming close to the aneurysm and covering the ICA proximal to the aneurysm, removal of the ACP was conducted to secure the space for TAO. If the secured part of the ICA showed severe atherosclerotic wall changes and was considered a danger for TAO, the cervical ICA was secured for PVC.

\section{Clinical Characteristics and Radiological Findings}

The objective of the current study was to evaluate the anatomical features that facilitate predicting the difficulty of PVC by analyzing preoperative CTA data. The medical records were reviewed to obtain relevant clinical information, and all medical data were obtained using an electronic PACS. The CTA data were reviewed by neurosurgeons (T.N. and J.S.), and data were collected for the following variables: the distance between the ACP tip and the proximal aneurysmal neck, the distance between the posterior wall of the carotid "knee" of the clinoid ICA and the proximal aneurysmal neck, the presence of calcification at the ophthalmic segment of the ICA, and the maximum diameter of the aneurysm. The aneurysm size was defined as the maximum perpendicular distance of the dome from the neck plane. The ICA was divided into 7 segments as described by Bouthillier et al. ${ }^{6}$ The distance between the ACP tip and the proximal aneurysmal neck was measured on axial and sagittal CTA source images, and the real distance was calculated using the Pythagorean theorem (Fig. $1 \mathrm{~A}-\mathrm{C}) .^{7}$ The distance between the posterior wall of the carotid knee of the clinoidal segment of the ICA and the proximal neck of the aneurysm was calculated on the lateral view 3D-CTA images (Fig. 1D).

In the preoperative CTA images, we reviewed the Towne anteroposterior (AP) and true lateral views. Towne $\mathrm{AP}$ views were controlled at an angle of $30^{\circ}$ from the orbitomeatal line. We measured the following angles that reflected the tortuosity of the ICA (Fig. 1E-G): the angles between 1) a line perpendicular to the cranial base and the axis of the communicating ICA (angle A), 2) the axis of the communicating ICA and the ophthalmic ICA (angle $\mathrm{B}$ ) on the Towne AP view, and 3) the axis of the communicating ICA and the ophthalmic ICA (angle C) on the lateral view. The straightest course of each ICA segment was selected as the axis of ICA segments.

\section{Statistical Analysis}

The statistical analyses were conducted with the aid of commercially available statistics software (SPSS version 21.0, IBM Corp.). Univariate and multivariate analyses were both performed.

To examine the potential predictors of the difficulty of PVC, we compared the abovementioned variables between the PVC groups with (group A) and without (group B) ACL and/or cervical ICA exposure. The Mann-Whitney U-test was used for the quantitative variables to assess the differences in the estimated data between the 2 groups, whereas Fisher's exact test was used for the categorical variables. A multivariate analysis was then conducted using a binary logistic regression. The results were considered significant for probability values $<0.05$. Furthermore, a receiver operating characteristic (ROC) analysis was used to find the best cutoff distance for determining whether the distance affected the difficulty of securing the proximal ICA.

\section{Results}

\section{Patient Characteristics}

The clinical and radiological characteristics of the 65 patients who underwent PCoA aneurysm clipping are 

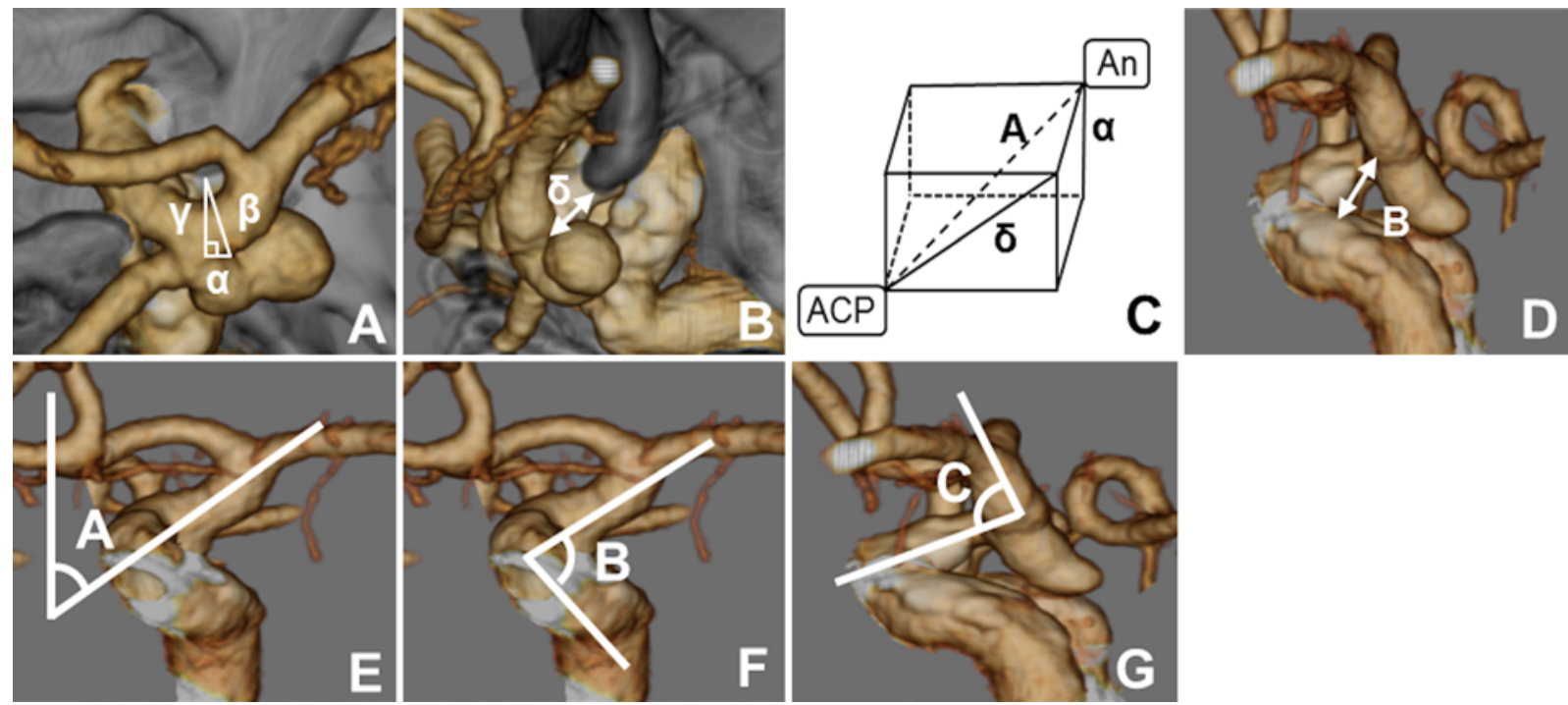

FIG. 1. 3D-CTA images obtained in a patient with a right ICA-PCoA aneurysm. A: Craniocaudal view of 3D-CTA without bone subtraction. Distance $\alpha=\left(\beta^{2}-\gamma^{2}\right)^{1 / 2}$, which indicates the distance between the tip of the ACP and the proximal neck of the aneurysm on the craniocaudal view. B: Lateral view of 3D-CTA without bone subtraction. The distance $\delta$ indicates the distance between the tip of the ACP and the proximal neck of the aneurysm on the lateral view. C: Distance $A=\left(\alpha^{2}+\delta^{2}\right)^{1 / 2}$, which is the real distance between the ACP and aneurysm neck (An) calculated with the Pythagorean theorem. D: Lateral view of 3D-CTA with bone subtraction. Distance $B$ indicates the distance between the posterior wall of the carotid knee and the proximal neck of the aneurysm. $\mathrm{E}$ : Angle $\mathrm{A}$ is measured between a line perpendicular to the cranial base and the axis of the communicating ICA on the Towne AP view. F: Angle $B$ is measured between the axis of the communicating ICA and the ophthalmic ICA on the Towne AP view. G: Angle $C$ is measured between the axis of the communicating ICA and the ophthalmic ICA segment on the lateral view.

summarized in Table 1. Patient age ranged from 30 to 92 years (mean \pm SD $67.2 \pm 12.1$ years) and 7 patients $(10.8 \%$ ) were male. Twenty microsurgical clipping procedures were performed for ruptured aneurysms (30.8\%), while 45 were performed for unruptured aneurysms $(69.2 \%)$. Based on an examination of the CTA data, the maximum diameter of the PCoA aneurysms ranged from 2.5 to $17.8 \mathrm{~mm}$ $(6.2 \pm 2.9 \mathrm{~mm})$. PVC was conducted in all patients and the vessels used for PVC and the procedures for securing these vessels were as follows: for 44 patients $(67.7 \%)$, the ophthalmic segment of the ICA was secured via the usual pterional approaches; for 6 patients (9.2\%), the ophthalmic segment of the ICAs was secured after ACLs; and for 15 patients (23.1\%), the cervical ICAs were secured.

\section{PVC Difficulties and Alternative Procedures}

Of the 65 patients included in this study, 21 patients (32.3\%) had characteristics that made it difficult to perform PVC with the usual pterional approach (Fig. 2). In 15 of these 21 patients, there was difficulty securing the ICA for PVC with TAOs because the ACP overhanging the ophthalmic segment of the intradural ICA obstructed the ability to secure a space for TAO (Fig. 2 left). In 13 of these 15 patients and 6 of the 50 patients in whom the ICA was secured for PVC with the usual pterional approach (Fig. 2 right), TAOs were difficult to achieve because of severe atherosclerotic wall changes in the ophthalmic segment of the intradural ICA proximal to the aneurysm (Fig. 3), and cervical ICAs were utilized for PVC.

In the 15 patients in whom ACP obstructed the space for TAO (Fig. 2 left), ACLs were performed in 13 of the
15 patients (Fig. 4). In 6 of these 13 patients the ophthalmic segment of the intradural ICA was used for PVC after ACL, in 6 patients the cervical ICA was used for PVC because of severe atherosclerotic wall changes in the ophthalmic segment of the intradural ICA proximal to the aneurysm, and in 1 patient the cervical ICA was used for

TABLE 1. Baseline characteristics of 65 patients who underwent surgical clipping for ICA-PCoA aneurysms

\begin{tabular}{lc}
\hline \multicolumn{1}{c}{ Characteristic } & Value \\
\hline Sex & \\
\hline Male & $7(10.8)$ \\
\hline Female & $58(89.2)$ \\
\hline Age, yrs & \\
\hline Aneurysm location & $35(53.8)$ \\
\hline Rt & $30(46.2)$ \\
\hline Lt & $6.2 \pm 2.9(2.5-17.8)$ \\
\hline Aneurysm size, mm & $20(30.8)$ \\
\hline Ruptured aneurysm & $45(69.2)$ \\
\hline Yes & \\
\hline No & $44(67.7)$ \\
\hline PVC procedure during clipping & $6(9.2)$ \\
\hline Intracranial ICA w/o ACL & $15(23.1)$ \\
\hline Intracranial ICA after ACL &
\end{tabular}

Values are presented as the number (\%) of patients or mean \pm SD (range). 


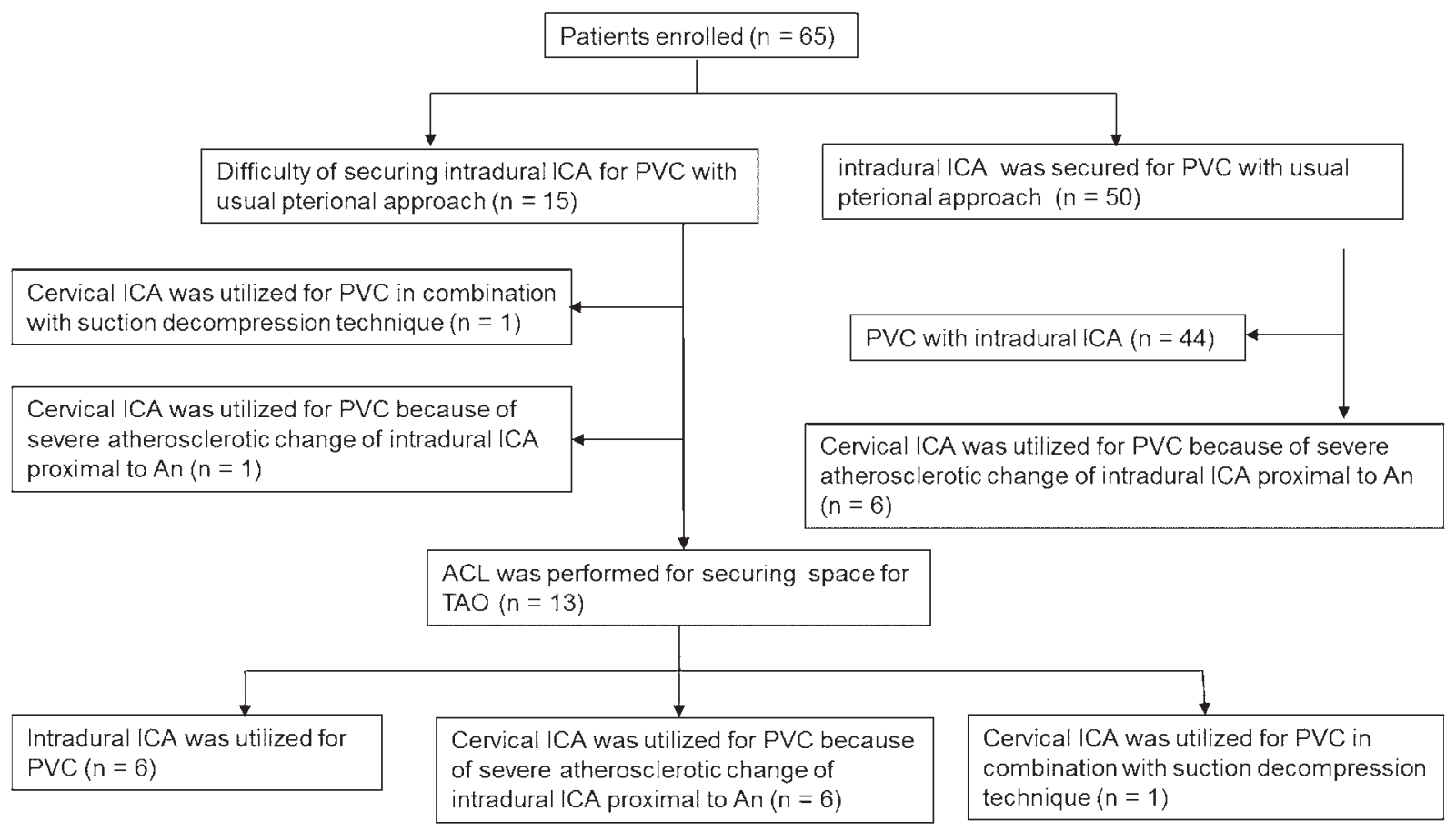

FIG. 2. Reasons for PVC difficulties and alternative procedures in 65 patients with $P C o A$ aneurysms (see Results section for a detailed description). An = aneurysm.

PVC in combination with retrograde suction decompression techniques (Fig. 2 bottom). ACL was not performed in 2 of the 15 patients. In 1 of these patients the cervical ICA was used for PVC in combination with retrograde suction decompression techniques, ${ }^{8}$ and in 1 patient the cervical ICA was used for PVC because of severe atherosclerotic wall changes in the ophthalmic segment of the intradural ICA proximal to the aneurysm (Fig. 2 left). In 6 of the 50 patients in whom the intradural ICA was secured for PVC with the usual pterional approach, the cervical ICA was used for PVC because of severe atherosclerotic wall changes in the ophthalmic segment of the intradural ICA proximal to the aneurysm (Fig. 2 right).

\section{Predictive Factors for Difficulty of PVC in Clipping Surgery for PCoA Aneurysms}

The mean, range, and $p$ value of each variable of the groups with (group A) and without (group B) difficulty of PVC are shown in Table 2. The results show that the 4 following variables were significantly different in the univariate analyses. First, the mean calculated distance between the proximal neck of the aneurysm and the ACP tip
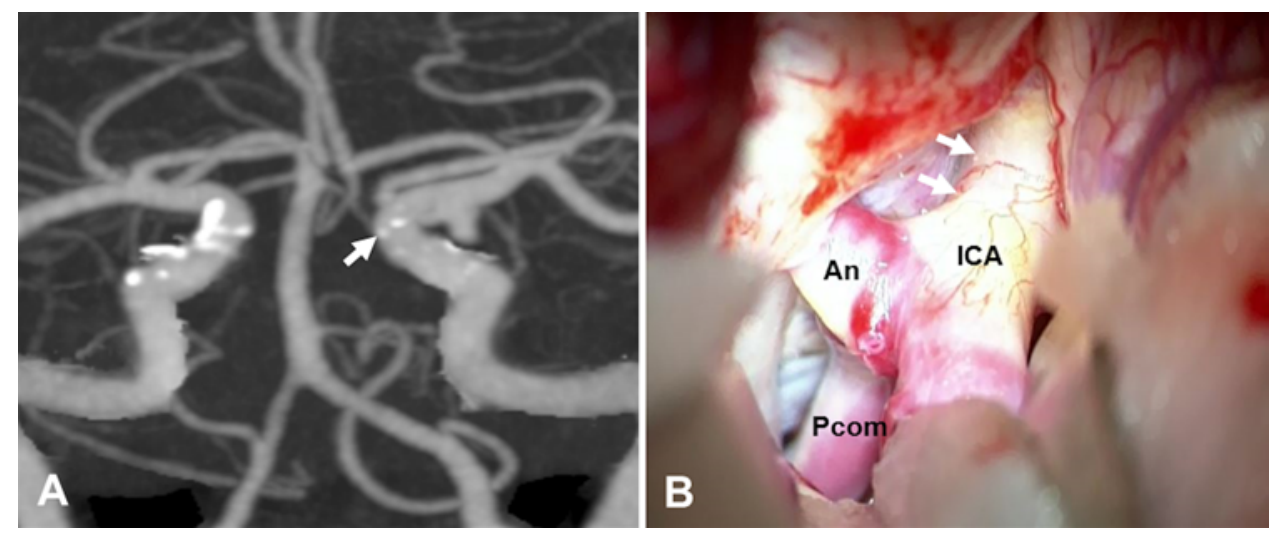

FIG. 3. A case of clipping of a left ICA-PCoA aneurysm A: A coronal maximum intensity projection (MIP) image from CTA. The solid white arrow indicates calcification of the left ophthalmic segment of the ICA. B: A microscopic view showing severe atherosclerotic wall changes (white arrows) in the ICA proximal to the aneurysm. In this case, the cervical ICA was utilized for PVC. Pcom = posterior communicating artery. 
was $4.7 \pm 1.5 \mathrm{~mm}$ (range $2.4-8.1 \mathrm{~mm}$ ) in group A, whereas in group B it was $7.2 \pm 2.5 \mathrm{~mm}$ (range $3.2-13.4 \mathrm{~mm}, \mathrm{p}<$ 0.001 ). Second, the mean calculated distance between the proximal aneurysmal neck and the posterior wall of the carotid knee of the clinoid ICA was $6.7 \pm 1.9 \mathrm{~mm}$ (range $3.9-10.2 \mathrm{~mm}$ ) in group A, whereas in group B it was $8.2 \pm$ $2.5 \mathrm{~mm}$ (range 3.6-14.1 mm, $\mathrm{p}=0.011$ ). Third, the mean maximum diameter of the aneurysm was $6.8 \pm 3.8 \mathrm{~mm}$ (range 3.2-17.8 mm) in group A, whereas in group B it was $5.5 \pm 2.0 \mathrm{~mm}$ (range $2.5-13.6, \mathrm{p}=0.039$ ). Fourth, the presence of calcification in the ophthalmic segment of the ICA on the preoperative CTAs was significantly different in the 2 groups (group A 57.1\% [12 of 21 patients] vs group B $4.5 \%$ [2 of 44 patients], $p<0.001$ ). In contrast, angles A, $\mathrm{B}$, and $\mathrm{C}$ showed no between-group differences.

The multivariate analysis using binary logistic regression revealed that the maximum diameter of the aneurysm $(p=0.041)$, the distance between the proximal neck of the aneurysm and the ACP tip ( $\mathrm{p}=0.002)$, and the presence of calcification in the ICA ophthalmic segment $(\mathrm{p}=$ 0.001 ) were significant predictive factors for the difficulty of PVC (Table 3).

\section{Predictive Factors for the Difficulty of Securing the Space Around the Intradural ICA for PVC in Clipping Surgery for PCoA Aneurysms}

Of the 65 patients enrolled in this study, the space for TAO was obstructed by the ACPs covering the ICAs proximal to the aneurysm in 15 patients. The abovementioned variables of distances and angles were compared between the groups with (group C) and without (group D) the difficulty of securing a space for TAO, and the 2 following variables were significantly different: 1) the distance between the proximal neck of the aneurysm and the ACP tip (mean $7.0 \pm 2.5 \mathrm{~mm}$, range $3.2-13.4 \mathrm{~mm}$ in group $\mathrm{C}$ vs $4.4 \pm 1.5 \mathrm{~mm}$, range $2.4-7.1 \mathrm{~mm}$ in group $\mathrm{D}, \mathrm{p}<0.001$ ) and 2) the calculated distance between the proximal aneurysmal neck and the posterior wall of the carotid knee of the clinoid ICA (mean $8.1 \pm 2.4 \mathrm{~mm}$, range $3.6-14.1 \mathrm{~mm}$ in group $C$ vs mean $6.7 \pm 2.0 \mathrm{~mm}$, range $3.9-10.2 \mathrm{~mm}$ in group $\mathrm{D}, \mathrm{p}=0.042$ ). An ROC curve analysis revealed that a distance between the proximal aneurysmal neck and the ACP tip of $\leq 5.4 \mathrm{~mm}$ was the best cutoff value for the difficulty of securing an intradural ICA proximal to the aneurysm (area under the curve 0.800 , cutoff value sensitivity $80.0 \%$, and specificity $80.0 \%$ ).

\section{Discussion}

The purpose of this study was to assess the factors that can be used to predict cases with difficulty attaining PVC in clipping surgery for PCoA aneurysms. In our series, based on intraoperative findings, 2 important factors were identified for the difficulty of PVC: whether an ACP covers the ICA at the proximal portion of the aneurysm and the presence versus the absence of atherosclerotic wall changes in the ICA proximal to the aneurysm. Based on preoperative CTA data, 3 predictive factors for the difficulty of PVC were identified: the maximum diameter of the aneurysm, the distance between the proximal aneurysmal neck and the ACP tip, and the presence of calcifica-
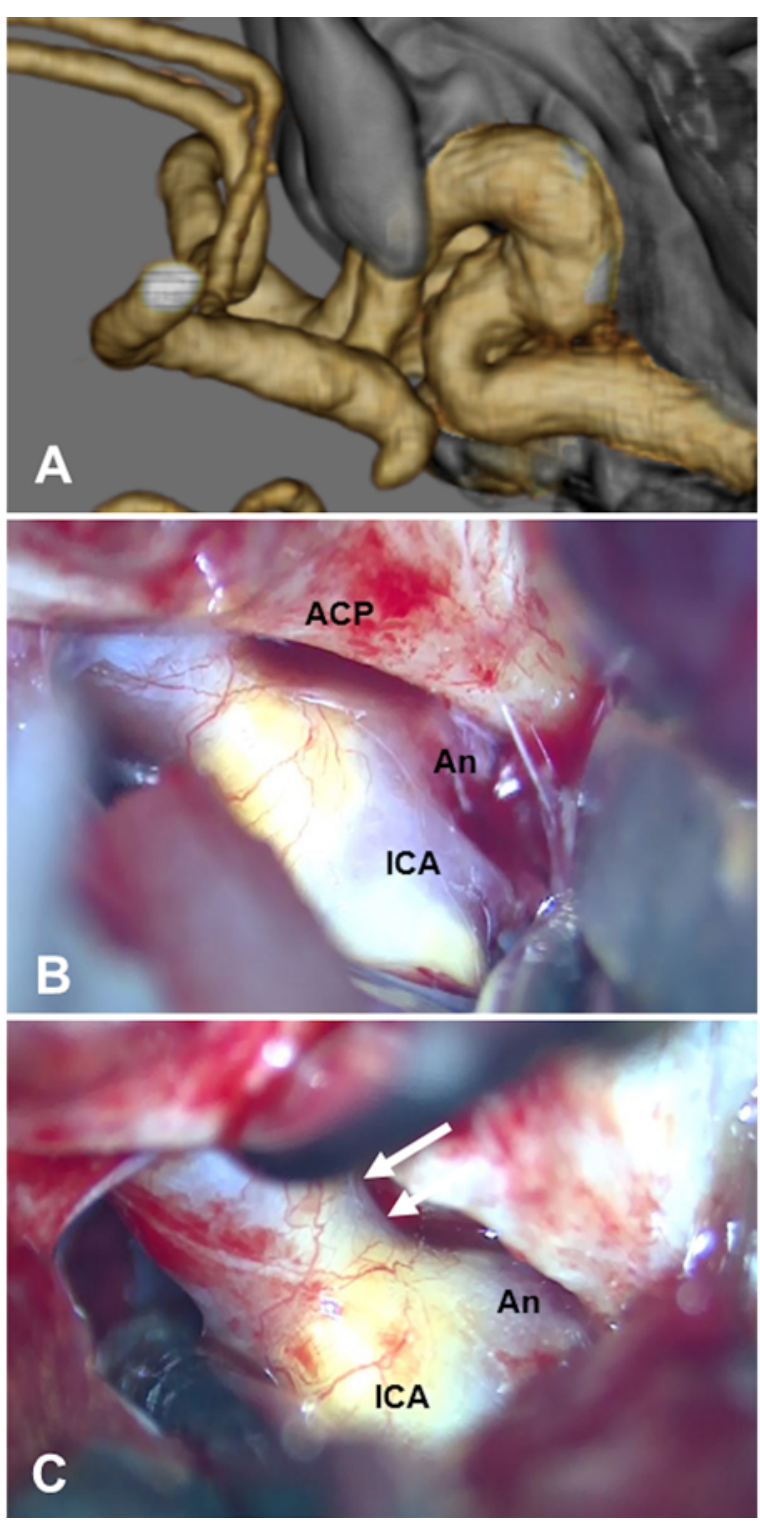

FIG. 4. A case of clipping of a right ICA-PCoA aneurysm. A: Preoperative 3D-CTA image. The proximal neck of the aneurysm is in close proximity to the ACP tip. The real distance between them calculated with the Pythagorean theorem is $3.3 \mathrm{~mm}$. B: A microscopic view before ACL was performed. There is no adequate space for TOC because the ACP is in close proximity to the aneurysm and overhangs the ophthalmic segment of the intradural ICA. C: A microscopic view after ACL was performed. In addition to exposure enhancement of the proximal neck of the aneurysm, adequate space for temporal artery occlusion was obtained (white arrows).

tion of the intradural ICA proximal to the aneurysm. A total of $32.3 \%$ of cases required ACL and/or exposure of the cervical ICA for PVC, which was a more frequent rate than expected.

In our series, cervical ICA securement for PVC was performed in 17 patients, including 2 patients with large aneurysms who underwent retrograde suction decompression. For a number of PCoA aneurysms, TAO is not necessary to achieve the safest and most ideal clipping. However, 
TABLE 2. Results of univariate analysis of factors for the necessity of ACL or exposure of the cervical ICA for PVC in ICA-PCoA aneurysm clipping

\begin{tabular}{|c|c|c|c|}
\hline Parameter & Group A & Group B & $\mathrm{p}$ Value \\
\hline Patients & $21(32.3)$ & $44(67.7)$ & \\
\hline Age, yrs & $70.0 \pm 11.2$ & $66.2 \pm 12.5$ & $0.407^{*}$ \\
\hline Sex & & & $0.531 \dagger$ \\
\hline Male & $3(14.3)$ & $4(9.1)$ & \\
\hline Female & $18(85.7)$ & $40(90.9)$ & \\
\hline Aneurysm size, mm & $6.8 \pm 3.8$ & $5.5 \pm 2.0$ & $0.039+$ \\
\hline Distance btwn ACP tip \& aneurysmal neck, mm & $4.7 \pm 1.5$ & $7.2 \pm 2.5$ & $<0.001 \dagger$ \\
\hline Distance btwn posterior wall of carotid knee \& aneurysmal neck, mm & $6.7 \pm 1.9$ & $8.2 \pm 2.5$ & $0.011 \dagger$ \\
\hline Angle $A,^{\circ}$ & $49.6 \pm 14.0$ & $50.0 \pm 14.9$ & $0.966 \dagger$ \\
\hline Angle $B,^{\circ}$ & $94.8 \pm 12.3$ & $94.9 \pm 20.2$ & $0.922 \dagger$ \\
\hline Angle $\mathrm{C}{ }^{\circ}$ & $61.8 \pm 14.2$ & $60.7 \pm 13.7$ & $0.637 \dagger$ \\
\hline ICA ophthalmic segment calcification on CTA & & & $<0.001^{*}$ \\
\hline Absent & $9(42.9)$ & $2(4.5)$ & \\
\hline Present & $12(57.1)$ & $42(95.5)$ & \\
\hline
\end{tabular}

in these patients, a high risk of IOR was predicted during surgery because of the co-occurrence of dense adhesions of the anterior choroidal arteries (AChAs) with the aneurysms and/or the presence of a thin aneurysm wall. The AChAs must be definitively dissected from the PCoA aneurysms to prevent devastating postoperative strokes with hemiparesis, hemisensory loss, and hemianopsia. TAO reduced the pressure within the aneurysm, which facilitated lessening the risk of IOR during clipping and dissecting the AChAs from the aneurysm. Of 15 patients with ACPs that overhung the ICAs proximal to the aneurysms and prevented the obtaining of adequate spaces for TAO in our series, removal of the ACPs enhanced the exposure of the ICAs proximal to the aneurysms and the creation of adequate spaces for TAO in all patients. The necessity of ACP removal has been emphasized previously in the description of surgical treatment of the ICA..$^{7,9-11}$ Nutik $^{10}$ reported in a cadaveric study that removal of the ACP permits visualization of approximately $6 \mathrm{~mm}$ more of the proximal ICA without entering the cavernous sinus. Andaluz et al. ${ }^{12}$ demonstrated in a cadaveric study that mean increases of $60 \%$ and $113 \%$ of the lateral and medial ophthalmic segments of the ICA, respectively, were observed with ACL.

The difficulty of securing an intradural ICA for TAO depends on the anatomical features, especially the relationship between the aneurysm and the ACP, which is widely variable in each patient. In our series, the short distance between the ACP tip and the aneurysmal neck was a robust factor for predicting the difficulty of securing spaces for TAO. Park et al. ${ }^{7}$ investigated the potential of predicting via preoperative radiological images whether ACL was needed in 94 cases of PCoA aneurysmal clipping. The authors noted that the distance between the ACP and aneurysmal neck calculated from the CTA data was shorter than $5.1 \mathrm{~mm}$ in the 6 cases requiring ACL. The authors also measured 2 angles on digital subtraction angiography (the same angles as A and B in our study), and angles $\mathrm{A}$ and $\mathrm{B}$ were significantly larger and smaller, respectively, in the ACL group than in the non-ACL group. In our series, these angles were not predictive for the difficulty of securing the space around the intradural ICA for TAO. The large and small angles of A and B, respectively, reflect the fact that the origin of the PCOA is located at the deeper side of the cranial base. However, an ophthalmic segment of the ICA coursing at the deeper side of the cranial base is not necessarily equal to the ACP overhanging the ICA proximal to the aneurysm. For example, the portion of ICA proximal to the aneurysm is not covered by the ACP when the ophthalmic segment is long and/or when the size of the ACP is relatively small. In our series, the short distance between the proximal aneurysmal neck and the posterior wall of the carotid knee of the clinoid ICA,

TABLE 3. Results of multivariate analysis using binary logistic regression for the necessity of $A C L$ and/or exposure of the cervical ICA for PVC in ICA-PCoA aneurysm clipping

\begin{tabular}{lccc}
\hline \multicolumn{1}{c}{ Variable } & OR & $95 \% \mathrm{Cl}$ & $\mathrm{p}$ Value \\
\hline Aneurysm max diameter & 1.640 & $1.020-2.635$ & 0.041 \\
\hline Distance btwn aneurysm proximal neck \& ACP tip & 0.271 & $0.119-0.617$ & 0.002 \\
\hline ICA ophthalmic segment calcification present & 50.690 & $4.705-546.084$ & 0.001 \\
\hline
\end{tabular}


which reflects the length of the ophthalmic segment, was predictive of the difficulty of securing the proximal ICA.

In our series, 13 patients revealed severe atherosclerotic wall changes in the ICAs at the proximal portion of the aneurysms as intraoperative findings, and the cervical ICAs were utilized for PVC. TAO using a parent artery with severe atherosclerotic wall changes is dangerous because the internal arterial wall is stretched with clip placement, which might result in occlusion or stenosis of the parent artery. In addition, a parent artery with atherosclerotic wall changes might be difficult to occlude. Of 13 patients with intraoperative findings of severe atherosclerotic wall changes in the ICAs at the proximal portion of the aneurysms, the preoperative CTA images revealed calcifications of the ophthalmic segment of the ICA in 12 patients, whereas calcifications were absent in 1 patient. Even if preoperative CTA images showed absent calcification at the ophthalmic segment of the ICA, the neurosurgeon should always be prepared to perform cervical ICA exposure in case difficulty attaining PVC is encountered due to atherosclerotic changes in the proximal ICA.

Technical advancements have significantly improved surgical and endovascular treatments of PCoA aneurysms. The coiling of PCoA aneurysms tends to be relatively uncomplicated compared with the coiling of aneurysms located in other parts of the anterior circulation. ${ }^{13}$ An international study of unruptured intracranial aneurysms found that there was no significant difference in mortality or the total prevalence of adverse outcomes at 1 year between the clipping and coiling cohorts. ${ }^{14}$ A subgroup analysis of elderly patients enrolled in an international subarachnoid hemorrhage aneurysm trial showed that the proportion of patients with ICA aneurysms, including PCoA aneurysms, who were independent in daily activities at 1 year was significantly greater in the endovascular cohort than the surgical cohort. ${ }^{15}$ However, the major drawback of endovascular embolization is that this method has a higher risk of recurrence ${ }^{16}$ and rehemorrhage ${ }^{17,18}$ than clipping surgery, especially for large ICA-PCoA aneurysms. ${ }^{17}$ ICA-PCoA aneurysms with an elongated fundus and aneurysms associated with a fetal PCoA may have better outcomes with surgical clipping in terms of the completeness of occlusion and the preservation of the PCoA. ${ }^{19}$ The decision regarding the treatment of ICA-PCoA aneurysms should be made by weighing the challenges involved in the treatment of each individual patient to reduce the risk of complications. In cases for which the difficulty of PVC and better outcome with coil embolization are predicted preoperatively, coil embolization may be an effective treatment alternative. Treatment options will also vary by treatment center depending on the experience of the surgeon and interventionalist. The prediction of the difficulty of establishing PVC may help in the process of making decisions between endovascular and surgical therapies for PCoA aneurysms.

\section{Conclusions}

A short distance between the proximal aneurysmal neck and the ACP tip and the presence of calcification of the ophthalmic segment of the intradural ICA were the robust factors for predicting the difficulty of attaining PVC in aneurysm patients. The current study is limited because it is a retrospective review of a relatively small case series from a single institution. Notwithstanding, the identification of cases with difficulty in attaining PVC preoperatively and the proposal of appropriate surgical tactics will be informative for neurosurgeons.

\section{References}

1. Elijovich L, Higashida RT, Lawton MT, et al. Predictors and outcomes of intraprocedural rupture in patients treated for ruptured intracranial aneurysms: the CARAT study. Stroke. 2008;39(5):1501-1506.

2. Leipzig TJ, Morgan J, Horner TG, et al. Analysis of intraoperative rupture in the surgical treatment of 1694 saccular aneurysms. Neurosurgery. 2005;56(3):455-468.

3. Wong JM, Ziewacz JE, Panchmatia JR, et al. Patterns in neurosurgical adverse events: endovascular neurosurgery. Neurosurg Focus. 2012;33(5):E14.

4. Batjer H, Samson D. Intraoperative aneurysmal rupture: incidence, outcome, and suggestions for surgical management. Neurosurgery. 1986;18(6):701-707.

5. Lawton MT, Du R. Effect of the neurosurgeon's surgical experience on outcomes from intraoperative aneurysmal rupture. Neurosurgery. 2005;57(1):9-15.

6. Bouthillier A, van Loveren HR, Keller JT. Segments of the internal carotid artery: a new classification. Neurosurgery. 1996;38(3):425-433.

7. Park SK, Shin YS, Lim YC, Chung J. Preoperative predictive value of the necessity for anterior clinoidectomy in posterior communicating artery aneurysm clipping. Neurosurgery. 2009;65(2):281-286.

8. Eliava SS, Filatov YM, Yakovlev SB, et al. Results of microsurgical treatment of large and giant ICA aneurysms using the retrograde suction decompression (RSD) technique: series of 92 patients. World Neurosurg. 2010;73(6):683-687.

9. Heros RC, Nelson PB, Ojemann RG, et al. Large and giant paraclinoid aneurysms: surgical techniques, complications, and results. Neurosurgery. 1983;12(2):153-163.

10. Nutik SL. Removal of the anterior clinoid process for exposure of the proximal intracranial carotid artery. J Neurosurg. 1988;69(4):529-534.

11. Yasargil MG, Gasser JC, Hodosh RM, Rankin TV. Carotidophthalmic aneurysms: direct microsurgical approach. Surg Neurol. 1977;8(3):155-165.

12. Andaluz N, Beretta F, Bernucci C, et al. Evidence for the improved exposure of the ophthalmic segment of the internal carotid artery after anterior clinoidectomy: morphometric analysis. Acta Neurochir (Wien). 2006;148(9):971-976.

13. Golshani K, Ferrell A, Zomorodi A, et al. A review of the management of posterior communicating artery aneurysms in the modern era. Surg Neurol Int. 2010;1:88.

14. Wiebers DO, Whisnant JP, Huston J III, et al. Unruptured intracranial aneurysms: natural history, clinical outcome, and risks of surgical and endovascular treatment. Lancet. 2003;362(9378):103-110.

15. Ryttlefors M, Enblad P, Kerr RS, Molyneux AJ. International subarachnoid aneurysm trial of neurosurgical clipping versus endovascular coiling: subgroup analysis of 278 elderly patients. Stroke. 2008;39(10):2720-2726.

16. Raymond J, Guilbert F, Weill A, et al. Long-term angiographic recurrences after selective endovascular treatment of aneurysms with detachable coils. Stroke. 2003;34(6):1398-1403.

17. Aikawa H, Kazwkawa K, Nagata S, et al. Rebleeding after endovascular embolization of ruptured cerebral aneurysms. Neurol Med Chir. 2007;47:439-445.

18. Johnston SC, Dowd CF, Higashida RT, et al. Predictors of rehemorrhage after treatment of ruptured intracranial aneu- 
rysms: the Cerebral Aneurysm Rerupture After Treatment (CARAT) study. Stroke. 2008;39(1):120-125.

19. Zada G, Breault J, Liu CY, et al. Internal carotid artery aneurysms occurring at the origin of fetal variant posterior cerebral arteries: surgical and endovascular experience. $\mathrm{Neu}$ rosurgery. 2008;63(1)(suppl 1):ONS55-ONS62.

\section{Disclosures}

The authors report no conflict of interest concerning the materials or methods used in this study or the findings specified in this paper.

\section{Author Contributions}

Conception and design: Niibo. Acquisition of data: Niibo, Takizawa, Sakurai, Takebayashi, Koizumi, T Kobayashi, R Kobayashi, Kuris, Gotou, Tsuchiya. Analysis and interpretation of data: Niibo. Drafting the article: Niibo. Study supervision: Kamiyama.

\section{Correspondence}

Takeya Niibo: Asahikawa Red Cross Hospital, Asahikawa, Hokkaidou, Japan.takeya.niibo@gmail.com. 